\title{
Apixaban and Rosuvastatin Pharmacokinetics in Nonalcoholic Fatty Liver Disease
}

\author{
Rommel G. Tirona, Zahra Kassam, Ruth Strapp, Mala Ramu, Catherine Zhu, Melissa Liu, \\ Ute I. Schwarz, Richard B. Kim, Bandar Al-Judaibi, and Melanie D. Beaton
}

Department of Physiology and Pharmacology (R.G.T., C.Z., U.I.S, R.B.K.), Division of Clinical Pharmacology, Department of Medicine (R.G.T., C.Z., M.L., U.I.S., R.B.K.), Department of Medical Imaging (Z.K.), Division of Gastroenterology, Department of Medicine (B.A.-J., M.D.B.), and Lawson Health Research Institute (R.G.T., Z.K., R.S., M.R., U.I.S., R.B.K., M.D.B.), University of Western Ontario, London, Ontario, Canada; and Department of Medicine, University of Rochester, Rochester, New York (B.A.-J.)

Received November 23, 2017; accepted February 19, 2018

\section{ABSTRACT}

There is little known about the impact of nonalcoholic fatty liver disease (NAFLD) on drug metabolism and transport. We examined the pharmacokinetics of oral apixaban $(2.5 \mathrm{mg})$ and rosuvastatin $(5 \mathrm{mg})$ when administered simultaneously in subjects with magnetic resonance imaging-confirmed NAFLD $(N=22)$ and healthy control subjects $(N=12)$. The area under the concentration-time curve to the last sampling time $\left(\mathrm{AUC}_{0-12}\right)$ values for apixaban were not different between control and NAFLD subjects $(671$ and $545 \mathrm{ng} / \mathrm{ml} \times$ hour, respectively; $P=0.15$ ). Similarly, the $A U C_{0-12}$ values for rosuvastatin did not differ between the control and NAFLD groups (25.4 and
$20.1 \mathrm{ng} / \mathrm{ml} \times$ hour, respectively; $\boldsymbol{P}=0.28$ ). Furthermore, hepatic fibrosis in NAFLD subjects was not associated with differences in apixaban or rosuvastatin pharmacokinetics. Decreased systemic exposures for both apixaban and rosuvastatin were associated with increased body weight $(P<0.001$ and $P<0.05$, respectively). In multivariable linear regression analyses, only participant weight but not NAFLD, age, or SLCO1B1/ABCG2/CYP3A5 genotypes, was associated with apixaban and rosuvastatin $A_{U C} C_{0-12}(P<0.001$ and $P=0.06$, respectively). NAFLD does not appear to affect the pharmacokinetics of apixaban or rosuvastatin.

\section{Introduction}

Nonalcoholic fatty liver disease (NAFLD) is the most common liver disease, affecting approximately $30 \%$ of adult North Americans (Sayiner et al., 2016). It is defined by hepatic steatosis, in the absence of significant alcohol consumption and other causes of hepatic fat accumulation (Ludwig et al., 1980). NAFLD encompasses both simple steatosis and nonalcoholic steatohepatitis (NASH), the latter being a more advanced stage of the disease involving liver inflammation, hepatocyte ballooning, and ultimately progressing to fibrosis (Brunt et al., 2011). NASH fibrosis is of particular concern as it is associated with increased liver-related and overall mortality (Dulai et al., 2017). There are no currently approved medications to treat NASH. Current disease management involves lifestyle modification and pharmacotherapies for common comorbidities such as hypertension, diabetes, and dyslipidemia (Rinella and Sanyal, 2016). A number of pharmacologic agents for NASH are being studied in clinical trials that aim to reverse the histologic features of the disease, particularly fibrosis (Rotman and Sanyal, 2017).

This work was supported by the Canadian Institutes of Health Research [Grant MOP-136909].

https://doi.org/10.1124/dmd.117.079624.
Despite the wide prevalence of NAFLD, it is remarkable that relatively little is known regarding how this disease influences drug disposition in humans. Few studies have evaluated the pharmacokinetics of drugs in NAFLD. In one study, antipyrine metabolic clearance was found to be reduced in NASH (Fiatarone et al., 1991), indicating lower overall cytochrome $\mathrm{P} 450$ (P450) activity for the drug that is metabolized by multiple P450 isoenzymes (Engel et al., 1996). With respect to changes to particular P450 enzymes, it has been compellingly established that in vivo hepatic CYP2E1 activity is increased in NASH, as revealed by chlorzoxazone phenotyping (Emery et al., 2003; Orellana et al., 2006). In addition, we demonstrated that CYP3A activity is decreased in NAFLD using a combination of oral midazolam phenotyping and measurement of plasma $4 \beta$-hydroxycholesterol $(4 \beta \mathrm{HC})$, an endogenous metabolic biomarker (Woolsey et al., 2015). This reduced in vivo CYP3A activity in NAFLD was recently confirmed using a translational systems pharmacology approach (Krauss et al., 2017). Last, there are now several studies demonstrating that the systemic exposure of glucuronide metabolites of drugs, namely acetaminophen and morphine, are increased in NASH (Barshop et al., 2011; Canet et al., 2015; Ferslew et al., 2015). These findings were attributed to increased hepatocyte basolateral membrane expression of multidrug resistance protein (MRP) 3 and hepatocellular internalization of canalicular MRP2 (Hardwick et al., 2011; Canet et al., 2015). Taken together, there remains

ABBREVIATIONS: A, apical; AUC, area under the plasma concentration-time curve; $\mathrm{AUC}_{0-\infty}$, area under the concentration-time curve to infinity; $\mathrm{AUC}_{0-12}$, area under the plasma concentration-time curve to the last sampling time; $\mathrm{B}$, basolateral; $\mathrm{BCRP}$, breast cancer resistance protein; $\mathrm{CL}_{\text {renal }}$, renal clearance; $4 \beta \mathrm{HC}, 4 \beta$-hydroxycholesterol; $\mathrm{KHB}$, Krebs-Henseleit bicarbonate buffer; LC-MS/MS, liquid chromatography-tandem mass spectrometry; MR, magnetic resonance; MRI, magnetic resonance imaging; MRP, multidrug resistance protein; $m / z$, mass/charge ratio; NAFLD, nonalcoholic fatty liver disease; NASH, nonalcoholic steatohepatitis; OATP, organic anion-transporting polypeptide; P450, cytochrome P450; P-gp, P-glycoprotein; PNPLA3, patatin-like phosphatase domain containing 3; ROI, region of interest; $t_{1 / 2}$, half-life; $\mathrm{T}_{\max }$, time to maximum plasma concentration; $\mathrm{X}_{\text {urine, } 0-12}$, amount excreted in urine. 
a paucity of information on NAFLD-related changes in pharmacokinetics and the potential associated clinical impacts on drug efficacy and harms.

In this study, we compared the pharmacokinetics of apixaban, a direct-acting oral anticoagulant, and rosuvastatin, an 3-hydroxy3-methylglutaryl-CoA reductase inhibitor, in healthy subjects and patients with NAFLD. These two medications were chosen for evaluation because 1) they are commonly prescribed in the NAFLD population, affording a degree of practical relevance; and 2) they are drugs with well-characterized disposition pathways that may shed new insights to potential disease alterations in drug-metabolizing enzyme and transporter activity. Indeed, apixaban is eliminated through hepatic metabolism by CYP3A4/5, biliary excretion, renal excretion (glomerular filtration), and intestinal secretion (Raghavan et al., 2009; Wang et al., 2010). Apixaban is a substrate of the efflux transporters breast cancer resistance protein (BCRP) and P-glycoprotein (P-gp) (Zhang et al., 2013). Rosuvastatin is minimally metabolized and eliminated by biliary and renal secretion (Martin et al., 2003). It is a substrate of BCRP, MRP2, MRP4, and P-gp (Huang et al., 2006; Kitamura et al., 2008; Knauer et al., 2010) and the uptake transporters, organic anion transporting polypeptides (OATPs) 1B1, OATP1B3, OATP2B1, and sodium taurocholate cotransporting polypeptide in liver (Ho et al., 2006), as well as organic anion transporter 3 in kidney (Windass et al., 2007). Notably, rosuvastatin is used as an in vivo probe for drug interactions involving transporters, namely OATP1B1 and BCRP (Stopfer et al., 2016; Prueksaritanont et al., 2017).

\section{Materials and Methods}

Transepithelial Flux of Apixaban and Rosuvastatin in Polarized Caco-2 Cells

Caco-2 cells were obtained from American Type Culture Collection ATCC (Manassas, VA) and cultured in Dulbecco's modified Eagle's medium supplemented with $50 \mu \mathrm{g} / \mathrm{ml}$ streptomycin, $50 \mathrm{U} / \mathrm{ml}$ penicillin, L-glutamine $(2 \mathrm{mM})$, and $10 \%$ fetal bovine serum. For transepithelial flux experiments, cells were seeded on $0.4 \mu \mathrm{m}$ pore size, cell (12-well) culture inserts (VWR International, Mississauga, ON, Canada) at a density of 90,000 cells/well and grown for 14 days with media changes every 2 days. Prior to the start of a transport experiment, the media were removed from each compartment (apical and basal), washed, and replaced with Krebs-Henseleit bicarbonate buffer (KHB) (pH 7.4). Transport was initiated after the removal of KHB and replacement with $700 \mu$ l of KHB, including apixaban (22 $\mu \mathrm{M}$; Toronto Research Chemicals, Toronto, ON, Canada) or $\left[{ }^{3} \mathrm{H}\right]$ rosuvastatin (42 $\mu \mathrm{M}$; American Radiolabeled Chemicals, St. Louis, MO), in the donor compartment. Apixaban $(22 \mu \mathrm{M})$, rosuvastatin $(42 \mu \mathrm{M})$, fumitremorgin $\mathrm{C}$ $(5 \mu \mathrm{M})$, or verapamil $(50 \mu \mathrm{M})$ were included in both the apical and basolateral compartments in separate wells. Cells were incubated at $37^{\circ} \mathrm{C}$ with $5 \% \mathrm{CO}_{2}$ in a humidified environment, and $25-\mu 1$ aliquots were removed hourly from each compartment over 4 hours. The concentration of apixaban in samples was determined by liquid chromatography-tandem mass spectrometry (LC-MS/MS) as detailed below. The $\left[{ }^{3} \mathrm{H}\right]$ rosuvastatin concentration was determined by liquid scintillation spectrometry (TriCarb2900 TR; PerkinElmer, Waltham, MA).

\section{Pharmacokinetic Study Subjects}

Healthy individuals (control subjects) and NAFLD patients provided informed written consent to participate in this study, which was approved by the Human Subjects Research Ethics Board at the University of Western Ontario. Studies were conducted in accordance with the ethical standards of the Helsinki Declaration of 1975 (as revised in 1983). Participants were enrolled after the clinical assessment and evaluation of serum biochemistry and hematology. The inclusion criteria for the NAFLD group were age 18 years or older, and diagnosis based on the American Association for the Study of Liver Disease definition (Chalasani et al., 2012) and magnetic resonance (MR) imaging (MRI)-confirmed hepatic steatosis. Exclusion criteria were acute or chronic renal insufficiency, use of steatogenic medications, use of medications that inhibit or induce CYP3A,
OATPs, BCRP or P-gp and refusal to discontinue grapefruit juice or herbal medicines that are CYP3A/P-gp inducers 1 week prior to the pharmacokinetic study.

\section{MR Imaging and MR Elastography}

All imaging was performed with the patient in the supine position, on a 1.5tesla MRI scanner (Aera; Siemens, Erlangen, Germany).

MR Elastography Examination. A commercial pneumatic driver system (Resoundant; Mayo Clinic Foundation, Rochester, MN) was used to create mechanical waves that were introduced into the subject's liver. The passive driver was placed anteriorly over the patient's liver, centered at the level of the xiphoid process, and secured with an elastic belt. The passive driver was connected via a polyvinylchloride tube to the active driver, which was placed outside the scan room to induce $60-\mathrm{Hz}$ vibrations into the liver.

\section{Acquisition Sequences.}

MR Elastography. The standard MR elastography protocol for our institution was used. Initially, axial T2 HASTE (half Fourier single-shot turbo spin-echo) images of the liver were acquired during inspiration. Based on these images, four sections of liver were selected by the radiologist for the MR elastography portion of the study. Stiffness maps were generated using a standard inversion algorithm and were displayed with a $95 \%$ confidence map. Mean stiffness measurements were calculated based on overall stiffness from each of the four sections acquired, using a manually drawn region of interest (ROI). The ROI was placed only on areas within the $95 \%$ confidence map. The average stiffness for each liver section, as well as overall liver stiffness, was then calculated.

Fat Signal Fraction. Chemical shift gradient echo imaging is used commonly for liver fat quantification. Decreased signal intensity of the liver on out-of-phase images compared with in-phase images is characteristic of fatty infiltration. In this study, manual ROIs were placed over the liver on the acquired chemical shift images (in phase and out of phase). The fat signal fraction (FSF) was calculated by using the following formula, which takes into account the net signal in liver on out-of-phase images compared with in-phase images:

$$
F S F=(S I P-S O P) / 2(S I P)
$$

where SIP is the net hepatic signal on in-phase images and SOP is the net hepatic signal on out-of-phase images (Ma et al., 2009).

\section{Pharmacokinetic Study}

Within 1 month of MRI and MR elastography, subjects were admitted to the Centre for Clinical Investigation and Therapeutics (Lawson Health Research Institute, London, ON) after an overnight fast. Participants refrained from consumption of citrus juice or caffeine 1 day prior to the pharmacokinetic study. The discontinuation of treatment with statins was requested for those prescribed beginning 3 days prior to the study day. At baseline, a morning blood sample was provided for the measurement of $4 \beta \mathrm{HC}$ and the extraction of DNA for genetic analysis. Rosuvastatin $5 \mathrm{mg}$ (Crestor; AstraZeneca, Cambridge, UK) and apixaban $2.5 \mathrm{mg}$ (Eliquis; Bristol-Myers Squibb, New York, NY) was administered simultaneously by mouth in $100 \mathrm{ml}$ of water. Prior to dosing and at $0.5,1,2,4,6,8,10$, and 12 hours thereafter, blood samples were obtained through an indwelling venous catheter for ethylenediaminetetraacetic-acid plasma drug concentration analysis. Urine was collected in toto over three intervals $(0-4$, 4-8, and 8-12 hours) after dosing for the assessment of renal drug excretion. Approximately 4 and 10 hours after dosing, participants were provided with meals. Plasma and urine samples were stored at $-80^{\circ} \mathrm{C}$ until analysis.

\section{Apixaban and Rosuvastatin Analysis}

Plasma and urine samples were analyzed by LC-MS/MS for apixaban and rosuvastatin concentrations. Apixaban, rosuvastatin and d6-Rosuvastatin were obtained from Toronto Research Chemicals. The [13C, 2H7]-apixaban was purchased from Alsachim (Illkirch, Graffenstaden, France). Plasma $(50 \mu \mathrm{l})$ proteins were precipitated with acetonitrile $(150 \mu \mathrm{l})$ containing d6-rosuvastatin $(10 \mathrm{ng} / \mathrm{ml})$ and [13C,2H7]-apixaban $(50 \mathrm{ng} / \mathrm{ml})$ and were centrifuged at $13,000 \mathrm{~g}$ for 10 minutes at $4{ }^{\circ} \mathrm{C}$. The resulting supernatant $(175 \mu \mathrm{l})$ was dried in a SpeedVac at $60^{\circ} \mathrm{C}$ and reconstituted in $150 \mu \mathrm{l}(0.1 \%$ formic acid in water/acetonitrile; $75 \%$ l $25 \%$ ) for injection into the liquid chromatograph (Agilent 1200; Agilent 

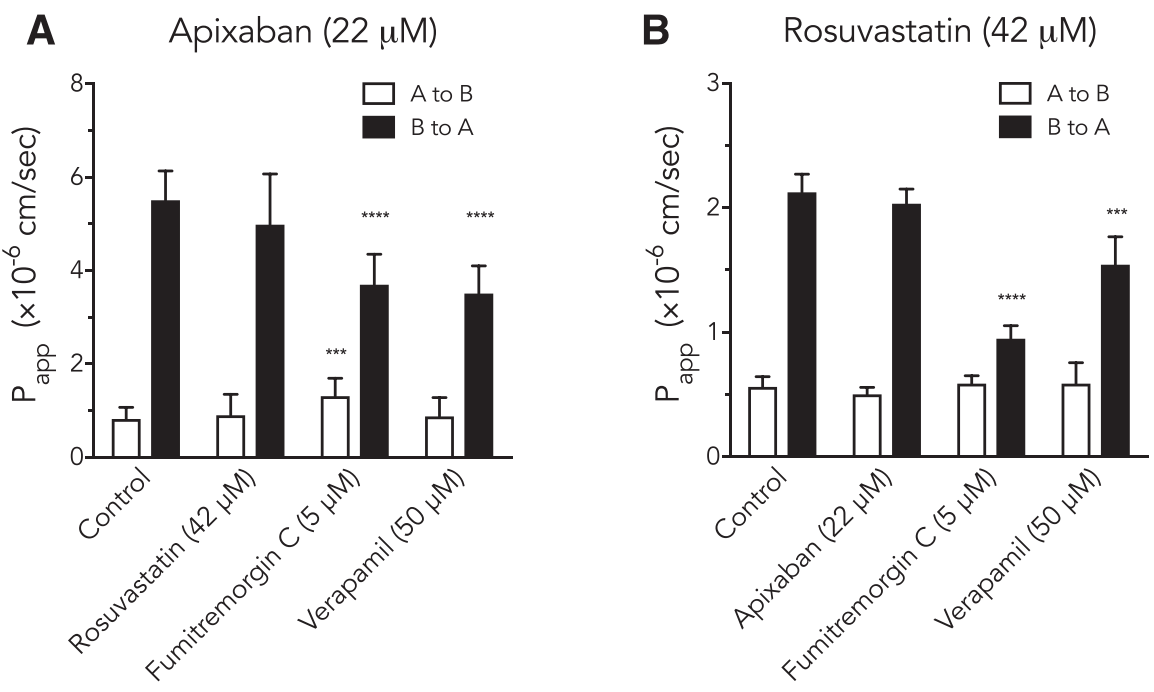

Fig. 1. Apixaban and rosuvastatin interactions in Caco-2 cells. Apixaban (A) and rosuvastatin (B) apparent permeabilities $\left(\mathrm{P}_{\text {app }}\right)$ across polarized Caco-2 cells in the A-to-B and B-to-A directions. Apixaban and rosuvastatin directional flux was monitored in the absence or presence of rosuvastatin, apixaban, fumitremorgin $\mathrm{C}$ (BCRP inhibitor), or verapamil (P-gp inhibitor) applied to both $\mathrm{B}$ and A compartments. Data are presented as the mean and S.D. $(N=3)$. $* * * P<0.001 ; * * * * P<0.0001$ when compared with control $\mathrm{P}_{\text {app }}$ using $t$ test.

Technologies, Santa Clara, CA). Analytes were separated on a Hypersil Gold C18 column $(50 \times 5 \mathrm{~mm}, 5 \mu \mathrm{m}$; ThermoFisher Scientific, San Jose, CA) under gradient elution with $0.1 \%$ formic acid in water and acetonitrile. With heated electrospray ionization, mass spectrometric detection (TSQ Vantage; ThermoFisher Scientific) was performed in positive mode with mass transitions $482.2 \rightarrow 258.1 \mathrm{mass} /$ charge ratio $(\mathrm{m} / \mathrm{z}), 488.1 \rightarrow 264.2 \mathrm{~m} / \mathrm{z}, 460.1 \rightarrow 443.3 \mathrm{~m} / \mathrm{z}$, and $468.1 \rightarrow 451.3 \mathrm{~m} / \mathrm{z}$ for rosuvastatin d6-rosuvastatin, apixaban, and [13C, 2H7]-apixaban, respectively. Calibration standards and quality control subjects were prepared in human plasma (BioreclamationIVT, Baltimore, $\mathrm{MD})$ and processed as described above. The between-run precision (CV percentage) and bias (percentage) values for quality control subjects for apixaban were $<14 \%$ and $<10 \%$, respectively, whereas those for rosuvastatin were $<12 \%$ and $10 \%$, respectively.

\section{$4 \beta \mathrm{HC}$ Analysis}

$4 \beta \mathrm{HC}$ concentration in plasma was determined by the picolinic acid derivatization and electrospray ionization LC-MS/MS method of Honda et al. (2008), as detailed in our previous report (Woolsey et al., 2016).

\section{Genotyping}

DNA was extracted from whole blood using the MagNA Pure System (Roche Diagnostics, Laval, QC, Canada). Single nucleotide polymorphism analysis was performed using TaqMan Allelic Discrimination Assays (Viia7; Applied Biosystems, Foster City, CA) for SLCO1B1 388A $>$ G (rs2306283), SLCO1B1 521T $>\mathrm{C}$ (rs4149056), ABCG2 421C $>\mathrm{A}$ (rs2231142), CYP3A4*22 (rs35599367), CYP3A5*3 (rs776746), and patatin-like phosphatase domain containing 3 (PNPLA3) I148M (rs738409). These genetic variants were chosen

TABLE 1

Participant characteristics

Values are the mean \pm S.D., unless otherwise indicated.

\begin{tabular}{|c|c|c|c|c|c|c|c|}
\hline & Control Group $(N=12)$ & NAFLD Group $(N=22)$ & $P$ Value* & $\begin{array}{l}\text { NAFLD-No Fibrosis Group } \\
\qquad(N=11)\end{array}$ & $P$ Value* & $\begin{array}{l}\text { NAFLD-Fibrosis Group } \\
\qquad(N=11)\end{array}$ & $P$ Value* \\
\hline Age $(y r)$ & $46.0 \pm 9.8$ & $51.3 \pm 12.4$ & 0.18 & $50.9 \pm 12.8$ & 0.32 & $51.7 \pm 12.5$ & 0.24 \\
\hline Sex, $n$ (female/male) & $10 / 2$ & $11 / 11$ & 0.056 & $7 / 4$ & 0.28 & $4 / 7$ & 0.021 \\
\hline Weight (kg) & $65.6 \pm 10.3$ & $99.9 \pm 21.9$ & $<0.001$ & $93.2 \pm 23.5$ & 0.003 & $106.6 \pm 18.8$ & $<0.001$ \\
\hline BMI & $23.4 \pm 2.5$ & $34.0 \pm 4.5$ & $<0.001$ & $32.4 \pm 3.9$ & $<0.001$ & $35.5 \pm 4.8$ & $<0.001$ \\
\hline Waist circumference $(\mathrm{cm})$ & $76.5 \pm 5.0$ & $111.4 \pm 17.9$ & $<0.001$ & $109.5 \pm 21.8$ & $<0.001$ & $113.1 \pm 14.3$ & $<0.001$ \\
\hline $\operatorname{ALT}(\mathrm{U} / \mathrm{l})$ & $16.5 \pm 7.5$ & $49.4 \pm 36.3$ & $<0.001$ & $49.1 \pm 32.1$ & 0.007 & $49.7 \pm 41.6$ & 0.024 \\
\hline AST (U/l) & $24.0 \pm 10.3$ & $33.1 \pm 15.5$ & 0.048 & $30.6 \pm 10.3$ & 0.14 & $35.6 \pm 19.6$ & 0.10 \\
\hline GGT (U/l) & $16.6 \pm 6.3$ & $52.1 \pm 40.8$ & $<0.001$ & $43.8 \pm 21.5$ & 0.002 & $60.5 \pm 53.6$ & 0.02 \\
\hline Alkaline phosphatase (U/l) & $61.3 \pm 14.3$ & $77.9 \pm 18.1$ & 0.006 & $79.1 \pm 16.8$ & 0.01 & $76.7 \pm 20.1$ & 0.05 \\
\hline Platelets $\left(\times 10^{9}\right.$ cells $\left./ 1\right)$ & $234 \pm 40$ & $262 \pm 75$ & 0.16 & $261 \pm 63$ & 0.23 & $262 \pm 89$ & 0.34 \\
\hline Serum creatinine $(\mu \mathrm{M})$ & $69.8 \pm 13.8$ & $67.8 \pm 12.1$ & 0.67 & $63.2 \pm 11.5$ & 0.23 & $71.9 \pm 11.7$ & 0.70 \\
\hline $\mathrm{HgA}_{1 \mathrm{c}}(\%)$ & $5.3 \pm 0.4$ & $6.3 \pm 0.9$ & $<0.001$ & $6.4 \pm 1.0$ & 0.004 & $6.2 \pm 0.8$ & 0.004 \\
\hline Diabetes & $0 / 12$ & $8 / 22$ & 0.017 & $2 / 11$ & 0.12 & $6 / 11$ & 0.003 \\
\hline Hypertension & $0 / 12$ & $10 / 22$ & 0.005 & $4 / 11$ & $<0.001$ & $6 / 11$ & 0.003 \\
\hline Dyslipidemia & $0 / 12$ & $11 / 22$ & 0.003 & $3 / 11$ & 0.052 & $8 / 11$ & $<0.001$ \\
\hline MR fat signal fraction & $-0.01 \pm 0.05$ & $0.27 \pm 0.08$ & $<0.001$ & $0.30 \pm 0.09$ & $<0.001$ & $0.24 \pm 0.08$ & $<0.001$ \\
\hline $4 \beta \mathrm{HC}(\mathrm{ng} / \mathrm{ml})$ & $16.9 \pm 3.2$ & $11.9 \pm 5.4$ & 0.007 & $13.4 \pm 5.9$ & 0.13 & $10.4 \pm 4.5$ & 0.002 \\
\hline \multicolumn{8}{|l|}{ Genotypes, $n$ (Frequency $\%)^{a}$} \\
\hline$S L C O 1 B 1388 \mathrm{~A}>\mathrm{G}$ & $7 / 5 / 0(21)$ & 8/9/5 (43) & 0.065 & $5 / 3 / 3(41)$ & 0.14 & $3 / 6 / 2(46)$ & 0.075 \\
\hline$S L C O 1 B 1521 \mathrm{~T}>\mathrm{C}$ & $8 / 4 / 0(17)$ & $15 / 7 / 0(16)$ & 0.935 & $7 / 4 / 0(18)$ & 0.89 & $8 / 3 / 0(14)$ & 0.78 \\
\hline$A B C G 2421 \mathrm{C}>\mathrm{A}$ & $7 / 4 / 1(25)$ & $20 / 2 / 0(5)$ & 0.012 & $10 / 1 / 0(5)$ & 0.054 & $10 / 1 / 0(5)$ & 0.054 \\
\hline$C Y P 3 A 4 * 22$ & $12 / 0 / 0(0)$ & 22/0/0 (0) & & $11 / 0 / 0(0)$ & & $11 / 0 / 0(0)$ & \\
\hline CYP $3 A 5 * 3$ & 8/3/1 (79) & 20/1/1 (93) & 0.086 & $9 / 1 / 1(86)$ & 0.52 & 11/0/0 (100) & 0.023 \\
\hline PNPLA3 I148M & $9 / 3 / 0(13)$ & $12 / 8 / 2(27)$ & 0.16 & $5 / 5 / 1(32)$ & 0.11 & $7 / 3 / 1(23)$ & 0.36 \\
\hline
\end{tabular}

ALT, alanine aminotransferase; AST, aspartate aminotransferase; BMI, body mass index; GGT, $\gamma$-glutamyltranspeptidase; $\mathrm{HgA}_{1 \mathrm{c}}$, glycated hemoglobin; Ref, carriers of reference allele; Var, variant allele.

${ }^{a}$ Genotypes are reported in the following pattern: Ref-Ref/Ref-Var/Var-Var.

*Two-tailed, $t$ test or $\chi^{2}$ test with comparison with control group. 
for evaluation as they are known to influence apixaban (Ueshima et al., 2017) or rosuvastatin (Keskitalo et al., 2009; DeGorter et al., 2013) pharmacokinetics. For PNPLA3, the genotype is associated with risk for NAFLD (Romeo et al., 2008).

\section{Pharmacokinetic Calculations}

Pharmacokinetic analysis was performed using model-independent methods. The area under the concentration-time curve to the last sampling time $\left(\mathrm{AUC}_{0-12}\right)$ was calculated using linear trapezoid method. The area under the concentrationtime curve to infinity $\left(\mathrm{AUC}_{0-\infty}\right)$ was calculated as the sum of $\mathrm{AUC}_{0-12}$ and $\mathrm{C}_{12} / \mathrm{k}$, where $\mathrm{C}_{12}$ is the last sampled concentration and $\mathrm{k}$ is the elimination rate constant obtained from the regressed slope of $1 n$-transformed terminal concentrations. Half-life $\left(t_{1 / 2}\right)$ was determined from $\ln 2 / \mathrm{k}$. $C_{\max }$ and time to $C_{\max }\left(\mathrm{T}_{\max }\right)$ were obtained directly from the observed results. Renal clearance $\left(\mathrm{CL}_{\text {renal }}\right)$ was calculated as the amount of drug excreted in urine from 0 to 12 hours $\left(\mathrm{X}_{\text {urine, } 0-12}\right)$ divided by $\mathrm{AUC}_{0-12}$.

\section{Statistical Analysis}

Comparisons of demographics, and serum biochemistry and pharmacokinetic parameters with subjects in the control group and NAFLD groups were performed using two-tailed, unpaired Student's $t$ test or $\chi^{2}$ test (Excel; Microsoft, Redmond, WA). Univariate associations between subject weight and drug $\mathrm{AUC}_{0-12}$ values were performed to obtain Pearson's correlation coefficients $(\rho)$ using GraphPad Prism (GraphPad Software, La Jolla, CA). Multivariable linear regression analyses were performed to determine the contribution of covariates (NAFLD status, age, weight, and $S L C O 1 B 1, A B C G 2$, and $C Y P 3 A 5$ genotypes) to apixaban and rosuvastatin $\mathrm{AUC}_{0-12}$ using SPSS (version 23; IBM Analytics, Armonk, NY). In multivariable linear regression analyses, we used an additive model for genotypes.

\section{Results}

Apixaban and Rosuvastatin Transport Interactions In Vitro. We assessed the possibility that simultaneous oral administration of apixaban and rosuvastatin may result in mutual pharmacokinetic interactions using the in vitro Caco-2 model. The transport of apixaban $(22 \mu \mathrm{M})$ across polarized Caco- 2 cells grown in the two-compartment configuration in either the apical (A)-to-basolateral (B) or B-to-A directions was not affected by the presence of rosuvastatin $(42 \mu \mathrm{M})$ applied to both compartments (Fig. 1A). In parallel experiments, the presence of fumitremorgin $\mathrm{C}(5 \mu \mathrm{M})$, a BCRP inhibitor, reduced both the A-to-B and B-to-A permeability of apixaban (Fig. 1A). Furthermore, the B-to-A flux of apixaban was attenuated by the presence of the P-gp inhibitor verapamil $(50 \mu \mathrm{M})$ (Fig. 1A). Our findings on the effects of BCRP and P-gp inhibitors on apixaban permeability in Caco-2 cells are similar to those reported previously (Zhang et al., 2013). Conversely, the presence of apixaban did not affect the transepithelial flux of rosuvastatin in both A-to-B and B-to-A directions (Fig. 1B). However, both fumitremorgin $\mathrm{C}$ and verapamil reduced the B-to-A transport of rosuvastatin (Fig. 1B). These results demonstrate a lack of mutual effects on bidirectional transport across Caco- 2 cells for apixaban and rosuvastatin.

Cohort Characteristics. Twelve healthy control and 22 NAFLD subjects were enrolled in the pharmacokinetic study. Detailed participant characteristics are presented in Table 1. MRI was used to detect and quantify the degree of hepatic steatosis for the inclusion of subjects into the control and NAFLD study groups. Fat signal fraction values of $\geq 0.1$ were used as a cutoff for the presence of hepatic steatosis and inclusion in the NAFLD group. The NAFLD cohort was segregated into NAFLD-no fibrosis $(N=11)$ and NAFLD-fibrosis $(N=11)$ subgroups by the presence of fibrosis as determined from liver biopsy findings or a positive MR elastography result (mean liver stiffness, $\geq 2.9 \mathrm{kPa}$ ). Participant age was similar between the control and NAFLD groups. Females tended to be over-represented in the control group (83\%) than the NAFLD group (50\%) $(P=0.056)$. Subjects were Caucasian with the exception of one African American participant in the control group. Total body weight, body mass index, waist circumference, and concentrations of serum biomarkers of liver function (alanine aminotransferase, aspartate aminotransferase, $\gamma$-glutamyltranspeptidase, and alkaline phosphatase) were significantly greater in the NAFLD group than the control group (Table 1). Serum creatinine levels were similar between groups. In the NAFLD group, diabetes, hypertension, and dyslipidemia were prevalent, with higher proportion of comorbidities in the NAFLD-fibrosis subgroup than in the NAFLD-no fibrosis subgroup. The allelic frequencies of the SLCO1B1 388A $>\mathrm{G}, S L C O 1 B 1521 \mathrm{~T}>\mathrm{C}$, and $C Y P 3 A 5 * 3$ polymorphisms were similar between the NAFLD group and control subjects. No $C Y P 3 A 4 * 22$ alleles were carried by any study participant. The decreased function $A B C G 2421 \mathrm{C}>\mathrm{A}$ polymorphism (Keskitalo et al., 2009) was found at a higher allelic frequency in the
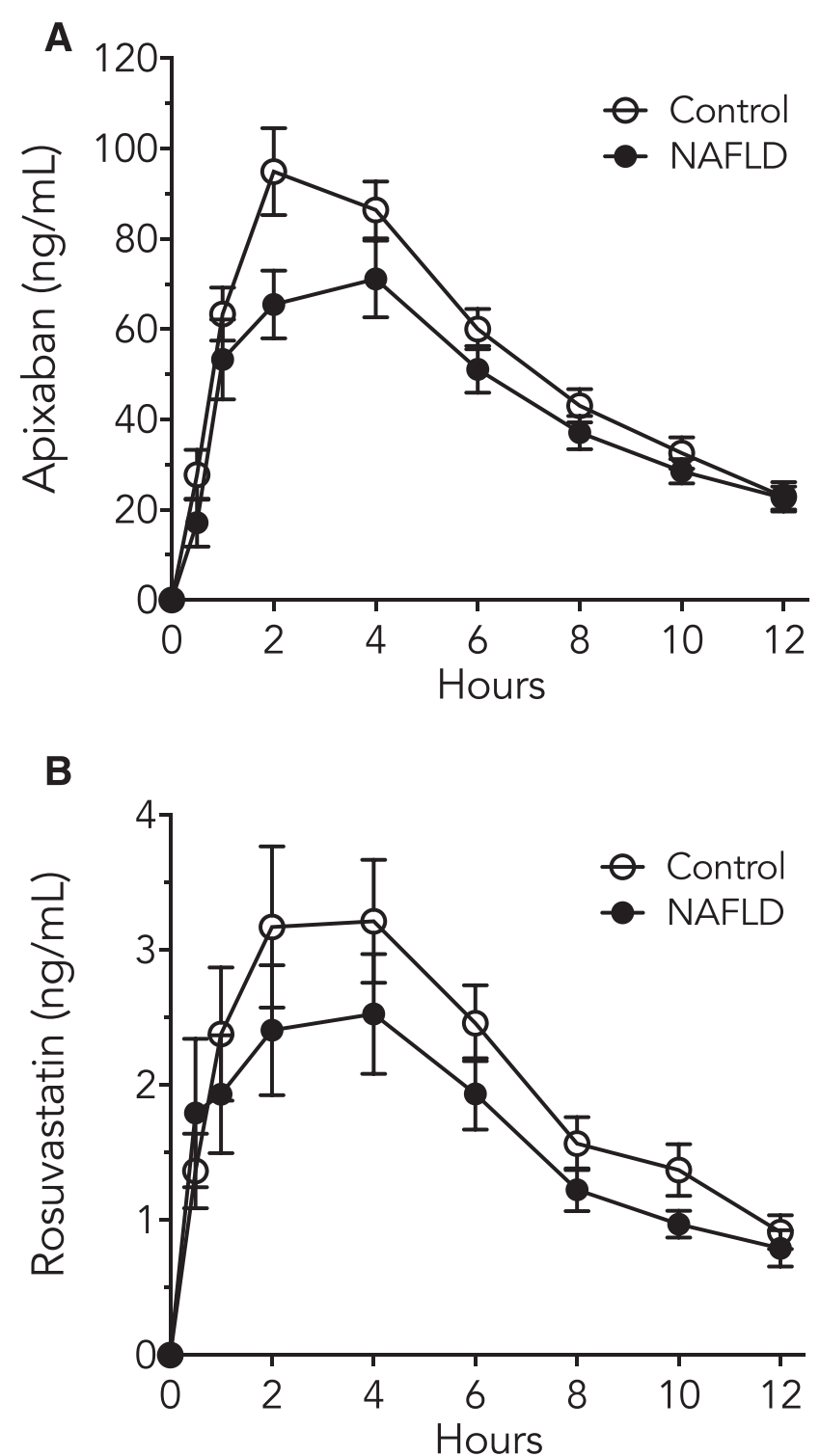

Fig. 2. Plasma concentration-time curves after simultaneous oral administration of apixaban $2.5 \mathrm{mg}$ (A) and rosuvastatin $5 \mathrm{mg}$ (B) in healthy control subjects (open circles; $N=12$ ) and patients with NAFLD (closed circles; $N=22$ ). Data are presented as the mean \pm S.E.M. 
TABLE 2

Apixaban pharmacokinetic parameters

Values are the mean \pm S.D., unless otherwise indicated.

\begin{tabular}{lcccccc}
\hline & $\begin{array}{c}\text { Control Group } \\
(N=12)\end{array}$ & $\begin{array}{c}\text { NAFLD Group } \\
(N=22)\end{array}$ & $P$ Value* & $\begin{array}{c}\text { NAFLD-No Fibrosis } \\
\text { Group }(N=11)\end{array}$ & $\begin{array}{c}P \text { Value* } \\
P \text { Value* }\end{array}$ & $\begin{array}{c}\text { NAFLD-Fibrosis } \\
\text { Group }(N=11)\end{array}$ \\
\hline $\mathrm{AUC}_{0-12}(\mathrm{ng} / \mathrm{ml} \times \mathrm{h})$ & $671 \pm 174$ & $545 \pm 265$ & 0.15 & $580 \pm 228$ & 0.30 & $511 \pm 304$ \\
$\mathrm{AUC}_{0-\infty}(\mathrm{ng} / \mathrm{ml} \times \mathrm{h})$ & $840 \pm 326$ & $715 \pm 351$ & 0.31 & $725 \pm 290$ & 0.38 & $705 \pm 417$ \\
$C_{\max }(\mathrm{ng} / \mathrm{ml})$ & $98 \pm 31$ & $78 \pm 44$ & 0.13 & $82 \pm 40$ & 0.15 \\
$\mathrm{~T}_{\max }(\mathrm{h})$ & $2.6 \pm 1.1$ & $2.7 \pm 1.3$ & 0.78 & $2.6 \pm 1.1$ & 0.96 & $74 \pm 49$ \\
$t_{1 / 2}(\mathrm{~h})$ & $4.5 \pm 1.5$ & $5.0 \pm 1.2$ & 0.34 & $4.5 \pm 0.5$ & 0.97 & $5.5 \pm 1.4$ \\
$\mathrm{X}_{\text {urine, } 0-12}(\mu \mathrm{g})$ & $611 \pm 148$ & $544 \pm 182$ & 0.26 & $527 \pm 176$ & 0.23 & $562 \pm 194$ \\
$\mathrm{CL}_{\text {renal }}(\mathrm{ml} / \mathrm{min})$ & $16.2 \pm 5.4$ & $19.6 \pm 9.1$ & 0.25 & $17.3 \pm 8.5$ & 0.71 & 0.12 \\
\hline
\end{tabular}

*Two-tailed, $t$ test or $\chi^{2}$ test with comparison with control group.

control group than in the NAFLD group $(P=0.012)$. Last, the PNPLA3 I148M variant, previously associated with hepatic steatosis and NASH risk (Romeo et al., 2008), was at similar prevalence between the control and NAFLD groups.

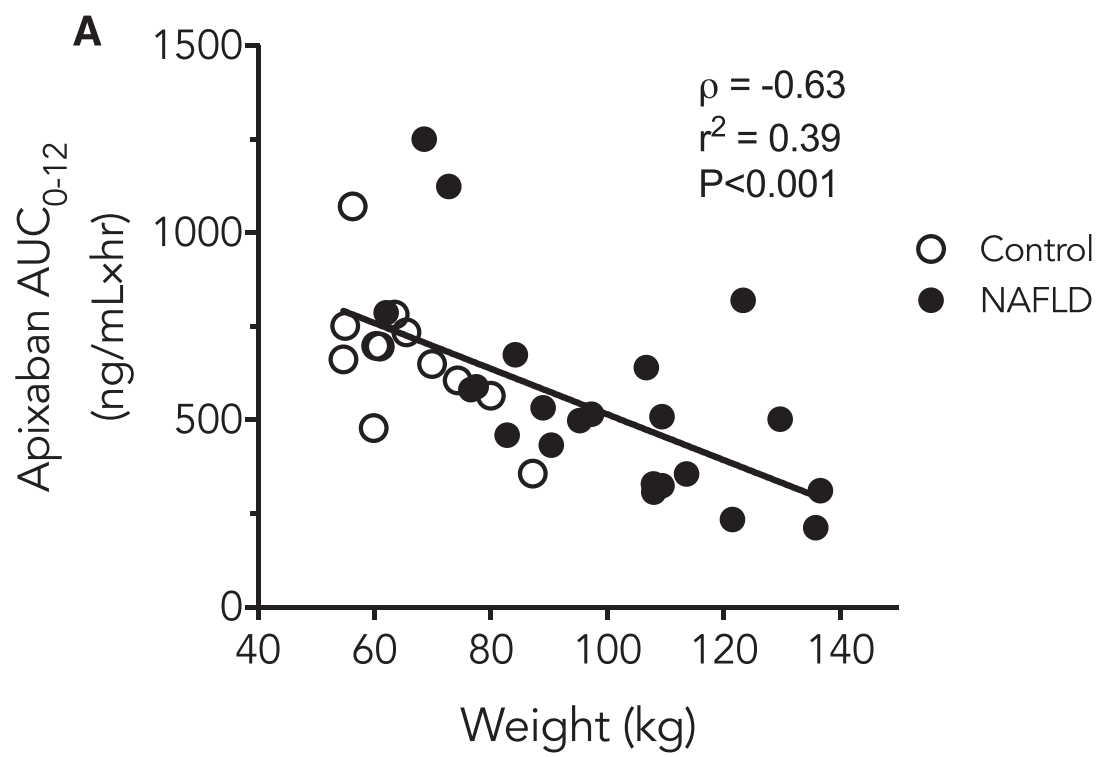

B

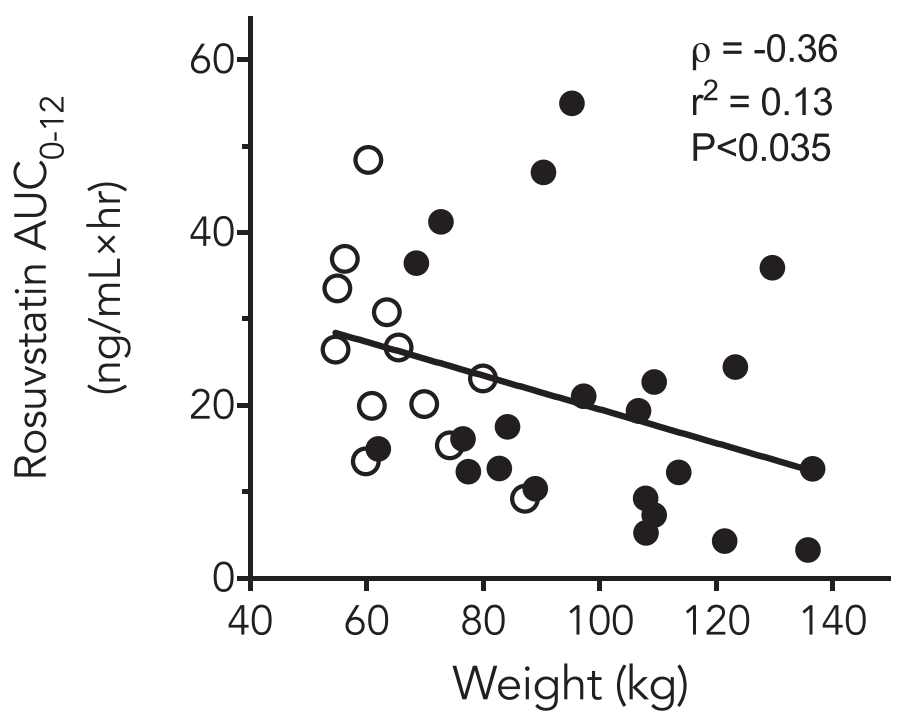

O Control

- NAFLD
Apixaban Pharmacokinetics. The mean plasma concentration-time profiles for apixaban in each study group are presented in Fig. 2A. Apixaban $\mathrm{AUC}_{0-12}, \mathrm{AUC}_{0-\infty}$, and $C_{\max }$ were not different between the control and NAFLD groups, although there was a trend toward lower
Fig. 3. Relationships between total body weight and $\mathrm{AUC}_{0-12}$ for apixaban (A) and rosuvastatin (B) in healthy control subjects (open circles; $N=12$ ) and patients with NAFLD (closed circles; $N=22$ ). Pearson's $\rho$ values, $r^{2}$ values, and $P$ values are noted. 
TABLE 3

Multivariable linear regression analysis of apixaban $\mathrm{AUC}_{0-12}$

\begin{tabular}{lcc}
\hline \multicolumn{1}{c}{ Variable } & Coefficient $(95 \% \mathrm{CI})$ & $P$ Value \\
\hline Constant & $1069(608,1529)$ & \\
Weight $(\mathrm{kg})$ & $-7.97(-11.76,-4.18)$ & $<0.001$ \\
Age $(\mathrm{yr})$ & $2.16(-4.21,8.52)$ & 0.49 \\
NAFLD & $146(-60,352)$ & 0.16 \\
$C Y P 3 A 5^{*} 3$ & $6.7(-142,155)$ & 0.93 \\
$A B C G 2421 C>A$ & $29.5(-139.8,198.8)$ & 0.72 \\
\hline
\end{tabular}

CI, confidence interval.

${ }^{a}$ Control $=0$, NAFLD $=1 ; R^{2}=0.46$.

values for these parameters in the NAFLD group $(P=0.15, P=0.31$, and $P=0.13$, respectively) (Table 2 ). $\mathrm{T}_{\max }, t_{1 / 2}, \mathrm{X}_{\text {urine, } 0-12}$, and $\mathrm{CL}_{\text {renal }}$ values were similar between the control the NAFLD groups (Table 2). Additionally, pharmacokinetic parameters were not different between either the NAFLD-no fibrosis subgroup or the NAFLD-fibrosis subgroup and the control group (Table 2). The apixaban $\mathrm{AUC}_{0-12}$ value was strongly correlated with total body weight with univariate $(P<0.001)$ (Fig. 3A) and multivariable linear regression analyses (Table 3). Neither NAFLD status, age, or $C Y P 3 A 5$ or $A B C G 2$ genotypes was associated with apixaban $\mathrm{AUC}_{0-12}$, as indicated by multivariable linear regression analyses (Table 3).

Rosuvastatin Pharmacokinetics. Mean plasma concentration results for rosuvastatin are shown in Fig. 2B. Rosuvastatin $\mathrm{AUC}_{0-12}$, $\mathrm{AUC}_{0-\infty}$, and $C_{\max }$ were similar between control and NAFLD groups (Table 4). Furthermore, $\mathrm{T}_{\max }, t_{1 / 2}, \mathrm{X}_{\text {urine, } 0-12}$, and $\mathrm{CL}_{\text {renal }}$ were similar among study groups (Table 4). Pharmacokinetic parameters were not different between the control and either the NAFLD-no fibrosis subgroup or the NAFLD-fibrosis subgroup (Table 4). With univariate comparison, there was a small but significant correlation between the rosuvastatin $\mathrm{AUC}_{0-12}$ value and total body weight $(P=0.035)$ (Fig. 3B) and a trend $(P=0.06)$ with multivariable linear regression analysis (Table 5). Rosuvastatin $\mathrm{AUC}_{0-12}$ was not associated with NAFLD status, age, or $S L C O 1 B 1$ or $A B C G 2$ genotypes with multivariable linear regression analysis (Table 5).

\section{Discussion}

Apixaban pharmacokinetics were not different between healthy control and NALFD subjects (Fig. 2A; Table 2). Previously, we found that CYP3A activity was moderately decreased in a separate NAFLD group (Woolsey et al., 2015). In the current cohort, we also observed reduced CYP3A activity in the NAFLD group, as suggested by examination of plasma $4 \beta \mathrm{HC}$ concentrations (Table 1 ). These decreased $4 \beta \mathrm{HC}$ concentrations were most pronounced in the NASH-fibrosis subgroup. Thus, it could have been anticipated that apixaban AUC would be greater in NAFLD subjects than in control subjects given that this anticoagulant is metabolized by CYP3A enzymes (Wang et al., 2010). It should be considered, however, that CYP3A-mediated metabolism is not a dominant elimination pathway for apixaban, as was evidenced by moderate increases (2-fold) in apixaban plasma AUC when coadministered with ketoconazole, a strong inhibitor of both CYP3A and P-gp (Frost et al., 2015). Approximately 27\% of apixaban clearance occurs through glomerular filtration in the kidney (Raghavan et al., 2009), and we observed that the renal elimination pathway was not different among the control and NAFLD groups whose serum creatinine concentrations were also similar. We found that the apixaban AUC strongly correlated with the total body weight of subjects, with lower systemic exposure seen with increasing mass (Fig. 3A). This result confirms previous observations at the extremes of body weight (Upreti et al., 2013), demonstrating that apixaban clearance follows allometric principles. We are unable to discern from the pharmacokinetic results whether NAFLD is associated with alterations in intestinal absorption or biliary and/or intestinal secretion of apixaban, which may have offset the observed reduction in CYP3A activity. Last, both $C Y P 3 A 5 * 3$ and $A B C G 2421 \mathrm{C}>\mathrm{A}$ genetic variations were not associated with apixaban AUC in this relatively small cohort despite that these genetic markers were recently linked to greater plasma trough concentrations in Japanese patients with atrial fibrillation (Ueshima et al., 2017). Although we did not find differences in apixaban plasma pharmacokinetics, additional focused studies are necessary to examine whether NAFLD may have specific effects on intestinal absorption and individual organ elimination pathways.

Interestingly, rosuvastatin systemic exposure was similar between the control and NAFLD groups (Fig. 2B; Table 4) despite there being reasons to expect otherwise. For example, NASH liver tissues have increased protein expression of OATP1B1 and reduced protein expression of OATP1B3 (Clarke et al., 2014b). Furthermore, the expression of MRP3, MRP4, MRP5, P-gp, and BCRP is increased in NASH (Hardwick et al., 2011). Although also upregulated, MRP2 is internalized from the hepatocyte canalicular membrane in NASH (Canet et al., 2015). Furthermore, there is reduced protein glycosylation of OATPs and MRP2 in NASH that may influence transporter function (Clarke et al., 2017). These changes in liver transporter expression appear to have an effect on the pharmacokinetics of glucuronide metabolites of acetaminophen and morphine (Barshop et al., 2011; Canet et al., 2015; Ferslew et al., 2015). Last, in a rodent model of $\mathrm{NASH}$, the systemic and liver disposition of simvastatin was altered, owing to the downregulation of hepatic Oatps (Clarke et al., 2014a). However, our current findings indicate that there is either a lack of functional changes in rosuvastatin transporters in NAFLD or that a complex interplay of altered activities of uptake and efflux transporters in different tissues results in no changes in rosuvastatin systemic

TABLE 4

Rosuvastatin pharmacokinetic parameters

Values are the mean \pm S.D., unless otherwise indicated.

\begin{tabular}{lcccccc}
\hline & $\begin{array}{c}\text { Control Group } \\
(N=12)\end{array}$ & $\begin{array}{c}\text { NAFLD Group } \\
(N=22)\end{array}$ & $P$ Value* & $\begin{array}{c}\text { NAFLD-No Fibrosis } \\
\text { Group }(N=11)\end{array}$ & $\begin{array}{c}P \text { Value* } \\
\text { Group }(N=11)\end{array}$ & $\begin{array}{c}\text { NAFLD-Fibrosis } \\
\text { G Value* }\end{array}$ \\
\hline $\mathrm{AUC}_{0-12}(\mathrm{ng} / \mathrm{ml} \times \mathrm{h})$ & $25.4 \pm 11.0$ & $20.1 \pm 14.3$ & 0.28 & $21.5 \pm 13.5$ & 0.46 & $18.6 \pm 15.7$ \\
$\mathrm{AUC}_{0-\infty}(\mathrm{ng} / \mathrm{ml} \times \mathrm{h})$ & $30.7 \pm 13.6$ & $25.4 \pm 17.0$ & 0.35 & $27.0 \pm 15.4$ & 0.58 & $23.7 \pm 19.2$ \\
$C_{\max }(\mathrm{ng} / \mathrm{ml})$ & $3.7 \pm 2.0$ & $3.0 \pm 2.6$ & 0.42 & $3.3 \pm 2.6$ & 0.70 & $2.8 \pm 2.7$ \\
$\mathrm{~T}_{\max }(\mathrm{h})$ & $3.9 \pm 2.0$ & $3.8 \pm 1.9$ & 0.86 & $3.7 \pm 2.1$ & 0.36 \\
$t_{1 / 2}(\mathrm{~h})$ & $4.4 \pm 1.2$ & $5.4 \pm 2.9$ & 0.21 & $4.8 \pm 1.4$ & 0.51 & $3.9 \pm 1.8$ \\
$\mathrm{X}_{\text {urine,0-12 }}(\mu \mathrm{g})$ & $257 \pm 107$ & $209 \pm 186$ & 0.34 & $244 \pm 230$ & 0.87 & $174 \pm 130$ \\
$\mathrm{CL}_{\text {renal }}(\mathrm{ml} / \mathrm{min})$ & $180 \pm 64$ & $178 \pm 69$ & 0.93 & $178 \pm 84$ & 0.95 & 0.11 \\
\hline
\end{tabular}

*Two-tailed, $t$ test or $\chi^{2}$ test with comparison with control group. 
TABLE 5

Multivariable linear regression analysis of rosuvastatin $\mathrm{AUC}_{0-12}$

\begin{tabular}{|c|c|c|}
\hline Variable & Coefficient $(95 \% \mathrm{CI})$ & $P$ Value \\
\hline Constant & $51(24,78)$ & \\
\hline Weight (kg) & $-0.23(-0.48,0.01)$ & 0.06 \\
\hline Age (years) & $-0.24(-0.64,0.16)$ & 0.23 \\
\hline $\mathrm{NAFLD}^{a}$ & $3.0(-10.5,16.5)$ & 0.65 \\
\hline$S L C O 1 B 1521 \mathrm{~T}>\mathrm{C}$ & $5.8(-3.9,15.6)$ & 0.23 \\
\hline$A B C G 2421 C>A$ & $-2.56(-12.8,7.7)$ & 0.61 \\
\hline
\end{tabular}

CI, confidence interval.

${ }^{a}$ Control $=0$, NAFLD $=1 ; R^{2}=0.23$.

exposure. In this regard, it can be considered that the efficiency of renal secretion (i.e., $\mathrm{CL}_{\text {renal }}$ ) of rosuvastatin is unaffected by NAFLD (Table 4). Moreover, the recovery of rosuvastatin in urine (i.e., $\mathrm{X}_{\text {urine,0-12 }}$ ) was similar between NAFLD subjects and control subjects (Table 4), indicating that NAFLD did not change the bioavailabilty or the relative contribution of liver and kidney in the overall elimination. However, it is not certain whether hepatocyte intracellular concentrations and intrinsic biliary clearance of rosuvastatin differs in NAFLD subjects, given that these may be poorly reflected by the plasma concentrations. The genetic polymorphisms in SLCO1B1 $(521 \mathrm{~T}>\mathrm{C})$ and $B C R P(421 \mathrm{C}>\mathrm{A})$ are associated with increased rosuvastatin plasma concentrations (Pasanen et al., 2007; Keskitalo et al., 2009; DeGorter et al., 2013). However, this was not observed herein and likely is a consequence of the relatively small cohort size. Other genetic markers that may be associated with rosuvastatin disposition were not examined. There was a modest correlation between rosuvastatin AUC and body weight (Fig. 3B). This result is in keeping with a pharmacokinetic study in children and adolescents that showed rosuvastatin oral clearance was associated with weight (Macpherson et al., 2016).

It is possible that the experimental strategy of simultaneous administration of apixaban and rosuvastatin may have masked the impacts of NAFLD on drug disposition. A pharmacokinetic drug-drug interaction between rosuvastatin and apixaban as a study complication was considered unlikely for a number of reasons. First, apixaban is not expected to inhibit rosuvastatin transporters, including OATPs and P-gp, at clinical doses (Zhang et al., 2013; Tsuruya et al., 2017). Moreover, rosuvastatin is not an inhibitor of CYP3A or P-gp (Martin et al., 2002; Sakaeda et al., 2006), proteins that impact apixaban pharmacokinetics. However, it remains a possibility that apixaban and rosuvastatin mutually interact with BCRP or MRP transporters. To assess potential interactions on these efflux transporters, we examined the transcellular flux of both apixaban and rosuvastatin in the presence and absence of the alternate medication in the in vitro Caco-2 cell model (Fig. 1). We found a lack of mutual interactions between apixaban and rosuvastatin on bidirectional flux across the Caco- 2 cells, which express BCRP, MRPs, and P-gp, lending support for the use of simultaneous drug administration in the pharmacokinetic study. Nevertheless, we used the lowest available commercial doses for apixaban $(2.5 \mathrm{mg})$ and rosuvastatin $(5 \mathrm{mg}$ ) both for safety reasons as well as for minimizing a potential drugdrug interaction. The drug interaction potential may be different at higher doses of apixaban and rosuvastatin.

MRI and MR elastography were used to quantify hepatic steatosis and fibrosis, respectively. Both techniques have been well validated against liver biopsy, which is currently considered the gold standard for assessment of the presence and severity of histologic features of NAFLD (Dulai et al., 2016). Whereas fibrosis can be accurately measured with elastography, assessment of hepatic inflammation (NASH) requires liver biopsy. In the absence of liver histology, we were therefore not able to distinguish precisely those NAFLD subjects with NASH but no fibrosis.
Consequently, it is possible that the NAFLD-no fibrosis subgroup may be composed of subjects with NASH in addition to those with only simple steatosis. Within the NAFLD-fibrosis subgroup, we are similarly unable to completely define those participants with NASH. As the presence of fibrosis is highly associated with steatohepatitis (McPherson et al., 2015), we expect that the majority of subjects in the NAFLDfibrosis subgroup have NASH. Indeed, liver stiffness as measured by MR elastography, has been proposed as a noninvasive method to detect NASH (Chen et al., 2011). In the NAFLD subgroup analyses, the pharmacokinetics of apixaban and rosuvastatin were not different between subjects without or with fibrosis in comparison with control subjects. We suspect that no pharmacokinetic differences would also be apparent if the NAFLD group were stratified into simple steatosis and NASH subgroups.

The findings of this study may inform the safety profiles of apixaban and rosuvastatin in the NAFLD population. As with other direct-acting anticoagulants (Ruff et al., 2015), higher bleeding risk with apixaban will likely associated with increased plasma concentrations. NAFLD does not appear to alter the plasma concentration-anticoagulation response effects of apixaban (Bos et al., 2017). Given that the mean apixaban exposure was not greater in NAFLD subjects compared with control subjects, the disease itself may not necessarily be a factor for determining bleeding risk or therapeutic dose. For rosuvastatin, increased plasma concentrations have been associated with a risk for severe myopathy (Jacobson, 2006); however, we found that systemic exposures were not greater in NAFLD. Although our results do not reveal whether rosuvastatin hepatocyte intracellular concentrations are affected by NAFLD, the use of statins in this population with low-grade transaminitis appears safe from a hepatotoxicity perspective (Bril et al., 2017). It is important to note that our findings for apixaban and rosuvastatin in NAFLD should not be extrapolated to more advanced stages of the disease, such as in NASH cirrhosis. Moreover, the current pharmacokinetic results do not permit conclusions to be drawn with respect to the influence of NAFLD on the pharmacodynamics of apixaban and rosuvastatin.

In conclusion, we demonstrate that the pharmacokinetics of apixaban and rosuvastatin are not affected by NAFLD. These findings provide additional insights into our understanding of pharmacological factors that may affect drug response and adverse effects in patients with this highly prevalent disease.

\section{Authorship Contributions}

Participated in research design: Tirona, Kassam, Kim, and Beaton.

Conducted experiments: Tirona, Kassam, Strapp, Ramu, Zhu, Liu, Schwarz, Al-Judaibi, and Beaton.

Performed data analysis: Tirona, Kassam, and Beaton.

Wrote or contributed to the writing of the manuscript: Tirona, Kassam, and Beaton.

\section{References}

Barshop NJ, Capparelli EV, Sirlin CB, Schwimmer JB, and Lavine JE (2011) Acetaminophen pharmacokinetics in children with nonalcoholic fatty liver disease. J Pediatr Gastroenterol Nutr 52:198-202.

Bos S, Potze W, Siddiqui MS, Boyett SL, Adelmeijer J, Daita K, Lisman T, and Sanyal AJ (2017) Changes of in vitro potency of anticoagulant drugs are similar between patients with cirrhosis due to alcohol or non-alcoholic fatty liver disease. Thromb Res 150:41-43.

Bril F, Portillo Sanchez P, Lomonaco R, Orsak B, Hecht J, Tio F, and Cusi K (2017) Liver safety of statins in prediabetes or T2DM and nonalcoholic steatohepatitis: post hoc analysis of a randomized trial. J Clin Endocrinol Metab 102:2950-2961.

Brunt EM, Kleiner DE, Wilson LA, Belt P, and Neuschwander-Tetri BA; NASH Clinical Research Network (CRN) (2011) Nonalcoholic fatty liver disease (NAFLD) activity score and the histopathologic diagnosis in NAFLD: distinct clinicopathologic meanings. Hepatology 53:810-820. Canet MJ, Merrell MD, Hardwick RN, Bataille AM, Campion SN, Ferreira DW, Xanthakos SA, Manautou JE, A-Kader HH, Erickson RP, et al. (2015) Altered regulation of hepatic efflux transporters disrupts acetaminophen disposition in pediatric nonalcoholic steatohepatitis. Drug Metab Dispos 43:829-835.

Chalasani N, Younossi Z, Lavine JE, Diehl AM, Brunt EM, Cusi K, Charlton M, and Sanyal AJ; American Gastroenterological Association; American Association for the Study of Liver 
Diseases; American College of Gastroenterology (2012) The diagnosis and management of nonalcoholic fatty liver disease: practice guideline by the American Gastroenterological Association, American Association for the Study of Liver Diseases, and American College of Gastroenterology. Gastroenterology 142:1592-1609.

Chen J, Talwalkar JA, Yin M, Glaser KJ, Sanderson SO, and Ehman RL (2011) Early detection of nonalcoholic steatohepatitis in patients with nonalcoholic fatty liver disease by using MR elastography. Radiology 259:749-756.

Clarke JD, Hardwick RN, Lake AD, Canet MJ, and Cherrington NJ (2014a) Experimental nonalcoholic steatohepatitis increases exposure to simvastatin hydroxy acid by decreasing hepatic organic anion transporting polypeptide expression. J Pharmacol Exp Ther 348:452-458.

Clarke JD, Hardwick RN, Lake AD, Lickteig AJ, Goedken MJ, Klaassen CD, and Cherrington NJ (2014b) Synergistic interaction between genetics and disease on pravastatin disposition. J Hepatol 61:139-147.

Clarke JD, Novak P, Lake AD, Hardwick RN, and Cherrington NJ (2017) Impaired N-linked glycosylation of uptake and efflux transporters in human non-alcoholic fatty liver disease. Liver Int 37:1074-1081.

DeGorter MK, Tirona RG, Schwarz UI, Choi YH, Dresser GK, Suskin N, Myers K, Zou G, Iwuchukwu O, Wei WQ, et al. (2013) Clinical and pharmacogenetic predictors of circulating atorvastatin and rosuvastatin concentrations in routine clinical care. Circ Cardiovasc Genet $\mathbf{6}$ 400-408.

Dulai PS, Singh S, Patel J, Soni M, Prokop LJ, Younossi Z, Sebastiani G, Ekstedt M, Hagstrom H, Nasr P, et al. (2017) Increased risk of mortality by fibrosis stage in nonalcoholic fatty liver disease: systematic review and meta-analysis. Hepatology 65:1557-1565.

Dulai PS, Sirlin CB, and Loomba R (2016) MRI and MRE for non-invasive quantitative assessment of hepatic steatosis and fibrosis in NAFLD and NASH: clinical trials to clinical practice. J Hepatol 65:1006-1016.

Emery MG, Fisher JM, Chien JY, Kharasch ED, Dellinger EP, Kowdley KV, and Thummel KE (2003) CYP2E1 activity before and after weight loss in morbidly obese subjects with nonalcoholic fatty liver disease. Hepatology 38:428-435.

Engel G, Hofmann U, Heidemann H, Cosme J, and Eichelbaum M (1996) Antipyrine as a probe for human oxidative drug metabolism: identification of the cytochrome P450 enzymes catalyzing 4-hydroxyantipyrine, 3-hydroxymethylantipyrine, and norantipyrine formation. Clin Pharmacol Ther 59:613-623.

Ferslew BC, Johnston CK, Tsakalozou E, Bridges AS, Paine MF, Jia W, Stewart PW, Barritt AS, IV, and Brouwer KL (2015) Altered morphine glucuronide and bile acid disposition in patients with nonalcoholic steatohepatitis. Clin Pharmacol Ther 97:419-427.

Fiatarone JR, Coverdale SA, Batey RG, and Farrell GC (1991) Non-alcoholic steatohepatitis: impaired antipyrine metabolism and hypertriglyceridaemia may be clues to its pathogenesis. J Gastroenterol Hepatol 6:585-590.

Frost CE, Byon W, Song Y, Wang J, Schuster AE, Boyd RA, Zhang D, Yu Z, Dias C, Shenker A, et al. (2015) Effect of ketoconazole and diltiazem on the pharmacokinetics of apixaban, an oral direct factor Xa inhibitor. Br J Clin Pharmacol 79:838-846.

Hardwick RN, Fisher CD, Canet MJ, Scheffer GL, and Cherrington NJ (2011) Variations in ATPbinding cassette transporter regulation during the progression of human nonalcoholic fatty liver disease. Drug Metab Dispos 39:2395-2402.

Ho RH, Tirona RG, Leake BF, Glaeser H, Lee W, Lemke CJ, Wang Y, and Kim RB (2006) Drug and bile acid transporters in rosuvastatin hepatic uptake: function, expression, and pharmacogenetics. Gastroenterology 130:1793-1806.

Honda A, Yamashita K, Miyazaki H, Shirai M, Ikegami T, Xu G, Numazawa M, Hara T, and Matsuzaki Y (2008) Highly sensitive analysis of sterol profiles in human serum by LC-ESIMS/MS. J Lipid Res 49:2063-2073.

Huang L, Wang Y, and Grimm S (2006) ATP-dependent transport of rosuvastatin in membrane vesicles expressing breast cancer resistance protein. Drug Metab Dispos 34:738-742.

Jacobson TA (2006) Statin safety: lessons from new drug applications for marketed statins. Am J Cardiol 97 (8A):44C-51C

Keskitalo JE, Zolk O, Fromm MF, Kurkinen KJ, Neuvonen PJ, and Niemi M (2009) ABCG2 polymorphism markedly affects the pharmacokinetics of atorvastatin and rosuvastatin. Clin Pharmacol Ther 86:197-203.

Kitamura S, Maeda K, Wang Y, and Sugiyama Y (2008) Involvement of multiple transporters in the hepatobiliary transport of rosuvastatin. Drug Metab Dispos 36:2014-2023.

Knauer MJ, Urquhart BL, Meyer zu Schwabedissen HE, Schwarz UI, Lemke CJ, Leake BF, Kim RB, and Tirona RG (2010) Human skeletal muscle drug transporters determine local exposure and toxicity of statins. Circ Res 106:297-306.

Krauss M, Hofmann U, Schafmayer C, Igel S, Schlender J, Mueller C, Brosch M, von Schoenfels W, Erhart W, Schuppert A, et al. (2017) Translational learning from clinical studies predicts drug pharmacokinetics across patient populations. NPJ Syst Biol Appl 3:11.

Ludwig J, Viggiano TR, McGill DB, and Oh BJ (1980) Nonalcoholic steatohepatitis: Mayo Clinic experiences with a hitherto unnamed disease. Mayo Clin Proc 55:434-438.

Ma X, Holalkere NS, Kambadakone R A, Mino-Kenudson M, Hahn PF, and Sahani DV (2009) Imagingbased quantification of hepatic fat: methods and clinical applications. Radiographics 29:1253-1277.

Macpherson M, Hamrén B, Braamskamp MJ, Kastelein JJ, Lundström T, and Martin PD (2016) Population pharmacokinetics of rosuvastatin in pediatric patients with heterozygous familia hypercholesterolemia. Eur J Clin Pharmacol 72:19-27.

Martin PD, Kemp J, Dane AL, Warwick MJ, and Schneck DW (2002) No effect of rosuvastatin on the pharmacokinetics of digoxin in healthy volunteers. J Clin Pharmacol 42:1352-1357.
Martin PD, Warwick MJ, Dane AL, Hill SJ, Giles PB, Phillips PJ, and Lenz E (2003) Metabolism, excretion, and pharmacokinetics of rosuvastatin in healthy adult male volunteers. Clin Ther $\mathbf{2 5}$ : 2822-2835.

McPherson S, Hardy T, Henderson E, Burt AD, Day CP, and Anstee QM (2015) Evidence of NAFLD progression from steatosis to fibrosing-steatohepatitis using paired biopsies: implications for prognosis and clinical management. J Hepatol 62:1148-1155.

Orellana M, Rodrigo R, Varela N, Araya J, Poniachik J, Csendes A, Smok G, and Videla LA (2006) Relationship between in vivo chlorzoxazone hydroxylation, hepatic cytochrome P450 $2 \mathrm{E} 1$ content and liver injury in obese non-alcoholic fatty liver disease patients. Hepatol Res 34: $57-63$.

Pasanen MK, Fredrikson H, Neuvonen PJ, and Niemi M (2007) Different effects of SLCO1B1 polymorphism on the pharmacokinetics of atorvastatin and rosuvastatin. Clin Pharmacol Ther 82:726-733

Prueksaritanont T, Tatosian DA, Chu X, Railkar R, Evers R, Chavez-Eng C, Lutz R, Zeng W, Yabut J, Chan GH, et al. (2017) Validation of a microdose probe drug cocktail for clinical drug interaction assessments for drug transporters and CYP3A. Clin Pharmacol Ther 101.519-530.

Raghavan N, Frost CE, Yu Z, He K, Zhang H, Humphreys WG, Pinto D, Chen S, Bonacorsi S, Wong PC, et al. (2009) Apixaban metabolism and pharmacokinetics after oral administration to humans. Drug Metab Dispos 37:74-81.

Rinella ME and Sanyal AJ (2016) Management of NAFLD: a stage-based approach. Nat Rev Gastroenterol Hepatol 13:196-205.

Romeo S, Kozlitina J, Xing C, Pertsemlidis A, Cox D, Pennacchio LA, Boerwinkle E, Cohen JC, and Hobbs HH (2008) Genetic variation in PNPLA3 confers susceptibility to nonalcoholic fatty liver disease. Nat Genet 40:1461-1465.

Rotman Y and Sanyal AJ (2017) Current and upcoming pharmacotherapy for non-alcoholic fatty liver disease. Gut 66:180-190.

Ruff CT, Giugliano RP, Braunwald E, Morrow DA, Murphy SA, Kuder JF, Deenadayalu N, Jarolim P, Betcher J, Shi M, et al. (2015) Association between edoxaban dose, concentration, anti-factor Xa activity, and outcomes: an analysis of data from the randomised, double-blind ENGAGE AF-TIMI 48 trial. Lancet 385:2288-2295.

Sakaeda T, Fujino H, Komoto C, Kakumoto M, Jin JS, Iwaki K, Nishiguchi K, Nakamura T, Okamura N, and Okumura K (2006) Effects of acid and lactone forms of eight HMG-CoA reductase inhibitors on CYP-mediated metabolism and MDR1-mediated transport. Pharm Res 23:506-512.

Sayiner M, Koenig A, Henry L, and Younossi ZM (2016) Epidemiology of nonalcoholic fatty liver disease and nonalcoholic steatohepatitis in the United States and the rest of the world. Clin Liver Dis 20:205-214.

Stopfer P, Giessmann T, Hohl K, Sharma A, Ishiguro N, Taub ME, Zimdahl-Gelling H, Gansser D, Wein M, Ebner T, et al. (2016) Pharmacokinetic evaluation of a drug transporter cocktail consisting of digoxin, furosemide, metformin, and rosuvastatin. Clin Pharmacol Ther 100: 259-267.

Tsuruya Y, Nakanishi T, Komori H, Wang X, Ishiguro N, Kito T, Ikukawa K, Kishimoto W, Ito S, Schaefer O, et al. (2017) Different involvement of OAT in renal disposition of oral anticoagulants rivaroxaban, dabigatran, and apixaban. J Pharm Sci 106:2524-2534.

Ueshima S, Hira D, Fujii R, Kimura Y, Tomitsuka C, Yamane T, Tabuchi Y, Ozawa T, Itoh H, Horie M, et al. (2017) Impact of ABCB1, ABCG2, and CYP3A5 polymorphisms on plasma trough concentrations of apixaban in Japanese patients with atrial fibrillation. Pharmacogenet Genomics 27:329-336.

Upreti VV, Wang J, Barrett YC, Byon W, Boyd RA, Pursley J, LaCreta FP, and Frost CE (2013) Effect of extremes of body weight on the pharmacokinetics, pharmacodynamics, safety and tolerability of apixaban in healthy subjects. Br J Clin Pharmacol 76:908-916.

Wang L, Zhang D, Raghavan N, Yao M, Ma L, Frost CE, Maxwell BD, Chen SY, He K, Goosen TC, et al. (2010) In vitro assessment of metabolic drug-drug interaction potential of apixaban through cytochrome $\mathrm{P} 450$ phenotyping, inhibition, and induction studies [published correction appears in Drug Metab Dispos (2010) 38:885]. Drug Metab Dispos 38:448-458.

Windass AS, Lowes S, Wang Y, and Brown CD (2007) The contribution of organic anion transporters OAT1 and OAT3 to the renal uptake of rosuvastatin. J Pharmacol Exp Ther 322: $1221-1227$.

Woolsey SJ, Beaton MD, Choi YH, Dresser GK, Gryn SE, Kim RB, and Tirona RG (2016) Relationships between endogenous plasma biomarkers of constitutive cytochrome P450 3A activity and single-time-point oral Midazolam Microdose phenotype in healthy subjects. Basic Clin Pharmacol Toxicol 118:284-291.

Woolsey SJ, Mansell SE, Kim RB, Tirona RG, and Beaton MD (2015) CYP3A activity and expression in nonalcoholic fatty liver disease. Drug Metab Dispos 43:1484-1490.

Zhang D, He K, Herbst JJ, Kolb J, Shou W, Wang L, Balimane PV, Han YH, Gan J, Frost CE, et al. (2013) Characterization of efflux transporters involved in distribution and disposition of apixaban. Drug Metab Dispos 41:827-835.

Address correspondence to: Dr. Melanie D. Beaton, Division of Gastroenterology, Department of Medicine, University of Western Ontario, London Health Sciences Centre, Room A10-223, 339 Windermere Road, London, ON N6A 5A5, Canada. E-mail: melanie.beaton@lhsc.on.ca 